\title{
To move or to sense? Incorporating somatosensory representation into striatal functions David Robbe ${ }^{1,2,3}$
}

\begin{abstract}
A long-standing hypothesis postulates that the striatum is essential for the concurrent selection of adaptive actions and repression of inappropriate alternatives. Here, classical and recent anatomical and physiological studies are reviewed to show that, in mammals, the striatum can detect discrete taskrelevant sensory stimuli and continuously track somatosensory information associated with the generation of simple movements and more complex actions. Rather than contributing to the immediate selection of actions, the striatum may monitor the sensorimotor state of animals by integrating somatosensory information and motor-related signals on a moment-by-moment basis. Such function could be critical for the progressive acquisition or updating of adaptive actions and the emergence of an embodied sense of time.
\end{abstract}

\author{
Addresses \\ ${ }^{1}$ Aix-Marseille University, Département de Biologie, Parc Scientifique de \\ Luminy, 13273 Marseille, France \\ ${ }^{2}$ INSERM-Institut National de la Santé et de la Recherche Médicale, \\ UMR 1249, Marseille, Parc Scientifique de Luminy, 13273 Marseille, \\ France \\ ${ }^{3}$ INMED-Institut de Neurobiologie de la Méditerranée, Parc Scientifique \\ de Luminy, 13273 Marseille, France
}

Corresponding author: Robbe, David (davidrobbe@gmail.com)

Current Opinion in Neurobiology 2018, 52:123-130

This review comes from a themed issue on Systems neuroscience Edited by Michael Long and Rosa Cossart

https://doi.org/10.1016/j.conb.2018.04.009

0959-4388/@ 2018 Elsevier Ltd. All rights reserved.

\section{Introduction}

The striatum is the main entry point of the basal ganglia (BG) and it is generally assumed that the dorsal region of striatum (DS) contributes to motor control [1]. The exact nature of this contribution is debated [2,3]. A long standing hypothesis is that the DS is critical for action selection [4,5]. This hypothesis is mainly based on two striking features of the anatomy and physiology of the mammalian BG. First, the output nuclei of the BG are mostly composed of GABAergic projection neurons that provide a tonic inhibition on the thalamocortical network and on brainstem motor regions [6-8]. Second, the GABAergic striatal projection neurons (SPNs), which represent more than $95 \%$ of the striatal neurons, can be divided in two distinct classes, on the basis of the BG nuclei they innervate and of the type of dopamine receptor they express [9]. Briefly, activation of so-called direct pathway SPN (dSPN) will disinhibit thalamocortical neurons and subcortical motor regions to ultimately favor movement generation while activation of so-called indirect pathway SPN (iSPN) will reinforce tonic inhibition on BG targets and repress movements generation [10]. The opposite modulatory power of the direct and indirect-pathways SPN (d/iSPN) on cortical and subcortical motor regions makes of the BG a potential system to select, through disinhibition [11], a given action from a set of competing possibilities [4]. Despite its popularity, there is still no direct satisfying evidence in support of this theory. In addition, it is not clear how the organization of the $B G$ inputs, which constantly provide sensory and motor information from the entire body, could be integrated in this theory. The objective of this short review is to suggest an alternative framework in which an important function of the DS is the continuous monitoring of the sensorimotor state of the animal.

In this review, the sensorimotor state of an animal refers to both somatosensory information and efference copies of descending motor commands [12]. Somatosensory information is derived from external sensory stimulation of different parts of the body (e.g. an air puff directed on the whiskers or the back of an animal) and active movements (e.g. locomotion triggers rhythmical sensory stimulation of the paws contacting the ground; proprioception). And by actions, we refer to an ordered sequence of movements (e.g. the act of turning left is composed of several movements [2]).

\section{Somatotopic organization of sensory and motor corticostriatal projections}

DS SPN receive excitatory projections mainly from the entire neocortex and a set of thalamic nuclei. Early tracing experiments revealed that specific regions of the cortex project to specific, often non-overlapping, regions of the striatum $[13,14]$. The topography of cortico-striatal connections roughly respects the rostro-caudal and dorsomedial positions of the cortical regions providing these inputs. For instance, in rats, the prelimbic region of the prefrontal cortex projects to the ventro-frontal regions of the striatum, the cingulate cortex projects toward the anterior dorsomedial regions of the striatum, and the barrel cortex projects to dorsolateral striatal regions 
[15]. Altogether, a large body of anatomical experiments in non-human primates, cats and rats, at different levels of the $\mathrm{BG}$, lead to the important concept that sensorimotor, associative and limbic information are treated in the striatum through segregated parallel circuits that constitute cortico-basal ganglia-thalamo-cortical loops [16-18].

In the $B G$, one of the main channels of information processing is dedicated to the treatment of sensory and motor information coming from cortex. It is located in the dorsolateral region of the striatum (DLS) in rodents and the putamen in primates. This channel is somatotopically organized $[4,17]$. For example, the regions of the cortices that trigger arm movements or represent sensory stimulation of the arm, project on a specific region of the striatum while cortical regions related to sensory and motor representation of the face project on a more ventral region [19-23]. Functional evidence of a somatotopic organization of the DLS is also suggested from a series of studies in which extracellular recordings of the spiking activity of DLS neurons were performed in awake freely moving rats while a thorough somatosensory examination (cutaneous touch, passive manipulation) of all accessible body parts (head, vibrissae, paw, chest, chin, snout, ear, shoulder, cheek pad, and trunk) was performed. About half of the DLS neurons increased sharply their firing rate in response to the selective stimulation of a given part of the body and neurons responding to a given part of the body tended to be located close in space in relative agreement with anatomical predictions [24-27]. Finally, the somatotopic organization of the DLS was also apparent in a study on the abnormal processing of information by the striatum, in which local injections of a selective inhibitor of fast-spiking interneurons in specific striatal subregions led to the appearance of episodic rest tremors of specific body parts [28].

When looking at rodent stereotaxic atlases, it is striking that the DS is one of the biggest undivided regions of the brain. It is possible that the cortico-striatal connectivity, despite its topographical organization, does not allow to delineate clear boundaries between different somatic regions. Indeed it was shown that corticostriatal projections originating from the whisker-related motor and sensory cortices do converge in the DLS, but these projections also display significant divergence throughout the DS [29-31]. But maybe the lack of DS subdivisions finds its origin in the difficulty to quantitatively compare, across animals and studies, the pattern of cortico-striatal projections using heterogeneous retrograde or anterograde tracers injections. Two recent studies in mice have used computational neuroanatomic approaches to quantify data from several hundreds of well-localized single, double and triple injections of anterograde tracers in the cortex of mice $\left[32^{\bullet \bullet}, 33^{\bullet \bullet}\right.$ ]. Several important conclusions could be drawn regarding the organization of corticostriatal connections carrying somatosensory information. First, based on the provenance of the cortical input, it was possible to subdivide the DLS in five subregions functionally related to specific body parts, namely the trunk, lower limbs, upper limb, inner mouth and outer mouth (Figure 1a). Second, projections from the whisker-related barrel cortex were detected in these five subregions and beyond. Third, when two cortical regions displayed strong reciprocal connections (e.g. forelimb motor and sensory cortices) their striatal projections strongly overlapped. Fourth, the delineation of somatotopic boundaries in the DLS does not reflect step-like pattern of projections: a functionally homogenous cortical region can also provide a diffuse arborization extending outside its main site of projection. Fifth, sensory and motor corticostriatal projections are not limited to the classically defined DLS but cover most of the DS, including socalled dorsomedial regions $\left[32^{\bullet \bullet}, 33^{\bullet \bullet}\right]$. Altogether these studies provided quantitative evidence in support of the somatotopical organization of the sensory and motor cortical input to the striatum (with the notable exception of whisker-related information) and revealed an unexpected prevalence of these input beyond the traditional dorsolateral regions they were classically confined to.

\section{Processing of somatosensory stimuli in the dorsolateral striatum}

In regard of the aforementioned wealth of anatomical data showing that the striatum is equipped to process somatosensory information, it is not well known what said processing consists of. This is partly due to the challenge of separating sensory and motor components associated with movement generation when working with behaving animals. To overcome this issue, several studies used anesthetized rodents and reported that striatal neurons respond to passive deflections of the whisker $\left[34,35,36^{\bullet \bullet}, 37\right]$. Noticeably, Reig and Silberberg [36 $\left.{ }^{\bullet \bullet}\right]$ performed patch clamp recording of DLS projection neurons in anesthetized mice and examined the response of these neurons to whisker air-puffs, which consisted in a depolarization of their membrane potential. The authors reported stronger and faster responses for contralateral stimulation compared to ipsilateral stimulation. Response amplitudes were stronger when whiskers were stimulated bilaterally, showing that SPN integrate sensory information coming from both sides of the body. Such integration was not present in the barrel cortex. While this study provided original knowledge on the integrative sensory capacity of the striatum, the anesthetized approach makes it difficult to conclude on the possible behavioral function of such sensory processing. Still, the integration of bilateral whisker-related information by dSPN was impaired in dopamine-depleted mice [ $\left[38^{\circ}\right]$, a particularly interesting result in the context of the well-known somatosensory abnormalities observed in Parkinsonian patients [39]. More recently, Sippy et al. $\left[40^{\circ \bullet}\right.$ ] directly investigated the role of the DLS in head-restrained mice trained to lick a reward spout in response to single whisker deflections. 




Somatotopic organization of the dorsolateral striatum and electrophysiological singature of somatosensory responses. (a) The dorsolateral striatum

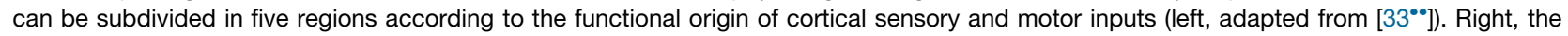
different regions of the mouse body are colors coded according to the striatal body map. (b) Schematic of the treadmill (top). Spiking raster plot of a DLS neuron with strong rhythmical modulation of the neuronal activity coordinated with the oscillatory dynamics of forelimb movements during treadmill locomotion (top). Green lines indicate treadmill onset, blue line goal time, orange arrow and dots indicate beginning and end of running (modified from [50"]). (c) Spiking raster plot (top) and peri-event histogram (bottom) of a DLS neuron, aligned to forepaw footfall, from [26].

The authors performed patch clamp recording and found that whisker-evoked depolarizations of SPN were stronger during 'hit' trials (in which the mouse successfully licked in response to the whisker deflection) compared to 'miss' trials. Whisker-evoked depolarization were composed of fast and slow components and the fast one was only expressed by dSPN. Brief optogenetic stimulation of the dSPN evoked licking. Altogether, this work shows for the first time that dSPN contribute to the expression of a conditioned motor response by signaling a predictive sensory stimuli.

\section{Somatosensory responses and action-related neuronal representations in the dorsal striatum}

If neuronal activity in the DLS can be modulated by somatosensory stimulation, either applied externally or resulting from movements, such modulations may be also apparent during the performance of motor tasks. While early electrophysiological studies in rodents and primates had reported modulations of firing occurring during movements (see also Panigrahi et al. [41]), a series of landmark articles have proposed that, after learning, DLS neurons mainly encode the initiation and ending of prolonged actions, in support of a role of the striatum in the gating of action [42-45]. Using cell-type specific calcium imaging in freely moving mice it was also shown that both dSPN and iSPN increased their activity just prior to lever press [46]. This work is now taken as a strong evidence supporting a role of $\mathrm{dSPN}$ in action selection while iSPN repress unwanted actions. Still, it remains possible that action initiation-related and termination-related activities are primarily driven by the somatosensory dynamics occurring around action initiation and termination (e.g. whisker stimulation, postural/limb adjustments). This possibility has not been directly tested but several recent works suggest that this interpretation should not be discarded. Coffey et al. [47 ${ }^{\bullet \bullet}$ ] used optrodes to identify 
dSPN and iSPN and found that about half of the recorded neurons in both populations increased their firing rate sharply in response to the passive stimulation of a given body part. In addition, it was shown in mice trained to perform a head-movements task that the firing rate of putative SPN is perfectly correlated with head-movements velocity [48]. This result confirmed previous studies in rats in which DLS neurons responding to passive head-movements were also correlated with head movements kinematics [49]. Similarly, in rats performing a learned running sequence on a treadmill, a fraction of the neurons fired synchronously with movements of the forelimb (Figure 1b) [50*], in agreement with previous reports of striatal neurons sensitive to passive and active forelimb movements (Figure 1c) [26,51]. Klaus et al. used calcium imaging to record the activity of ensemble of identified SPN in the DLS of mice exploring an open field. They found that similar exploratory actions (e.g. left turns) were associated with the activation of similar ensembles of SPNs that were not randomly located but spatially close [52], which could be accounted for by somatosensory responses to the movements that compose each action. Using a similar experimental setup, the activity of ensemble of dSPN and iSPN was shown to correlate with animals velocity, on a very slow speed range (from 0 to $10 \mathrm{~cm}$, a logarithmic scale was use to reveal the correlation) [53]. This encoding of locomotionrelated information (see also [54,55]) might be largely explained by an increased rate of somatosensory responses of striatal neurons when animals transitioned from resting to a variety of foraging and exploratory behaviors.

\section{Somatosensory responses in the striatum bring experimental challenges}

The fact that a large fraction of neurons in the DS (including identified $\mathrm{d} / \mathrm{iSPN}$ ) responds to a variety of somatosensory stimulation in behaving animals presents serious challenges when interpreting action-related neuronal patterns of activity. While it is tempting to assign causal interpretation to patterns of activity recorded in the striatum in terms of action generation/selection/initiation/ chunking/learning, the possibility that these patterns are 'merely' caused by movements and their associated sensory consequences is often overlooked [42-46,52,53,56]. Somatosensory responses in the striatum are related to the entire body, including the trunk, neck, paw, inner mouth and whiskers, which are difficult to track in behaving animals. Thus, an additional potential confound is that patterns of neuronal activity in the striatum may not be primarily related to an action of interest studied in a task (a lever-press, nose-poke, a bout of locomotion or even licking) but to secondary covert movements and sensory stimulation (whiskers, body postures, head or jaw movements) that precede, co-occur, or follow this action. These issues are especially important when investigating the neural bases of motor learning, which is associated with reorganization of somatosensory dynamics. To overcome these difficulties, a possibility is to use behavioral designs allowing a fair comparison between actions performed before and after learning. This approach revealed that striatal population activity recorded in untrained and trained animals performing a similar action (run back and forth on the belt of a motorized treadmill) displayed a similar over representation of the beginning and ending of that action (Figure 2) $\left[50^{\circ}\right]$. Thus, the representation of these specific action phases is likely to be primarily accounted for by the somatosensory sensibility of the DLS, rather than higher order processes such as learning or action selection/gating. A second experimental strategy is to complement imaging or electrophysiological recordings during spontaneous or conditioned behaviors with an exhaustive and quantitative somatosensory examination $\left[24,47^{\bullet \bullet}, 27\right]$. This is difficult to do in freely behaving animals. However, recording striatal activity while, for instance, an experimenter gently rotates the head of a mice toward the left, would allow to investigate to what extent the representation of a left turn action is accounted for by passive somatosensory responses.

\section{A role for striatal somatosensory responses in motor learning}

The high prevalence of somatosensory responses in the DS should not just be seen as a challenge but also as a key to the understanding of the behavioral function(s) of this brain region. The integration of somatosensory representation and efference copies of motor programs with reward prediction error signals could contribute to motor learning by linking a given sensorimotor state to an action that favored the consumption of rewards or prevented unpleasant/dangerous situations. This associative learning process could occur through bidirectional dopaminemediated synaptic plasticity of the cortico-striatal connections [9] that convey somatosensory information and efference copy of motor programs. The resulting changes in synaptic transmission strength could contribute to the formation of neuronal ensemble distributed across cortical and subcortical regions such as primary and secondary motor cortices, the striatum and the BG targets (thalamus and midbrain/brainstem motor nuclei). Such general hypothesis is in agreement with a recent study showing that learning a cue-guided motor sequence is associated with the strengthening of the excitatory connection between motor cortex and dorsolateral dSPN [57]. It is also supported by the fact that during performance of a simple voluntary action, the spiking activity of dorsolateral SPN is comodulated by movement-related and reward-related information [58]. The functional relevance of a sensorimotor tracking function of the dorsal striatum for learning purpose can be illustrated in the context of time estimation tasks. Rodents naturally develop embodied strategies when challenged in such tasks, independently if the time interval to estimate is short $[59,60]$ or long $\left[50^{\circ}\right]$ : they progressively refine, by 




(b)

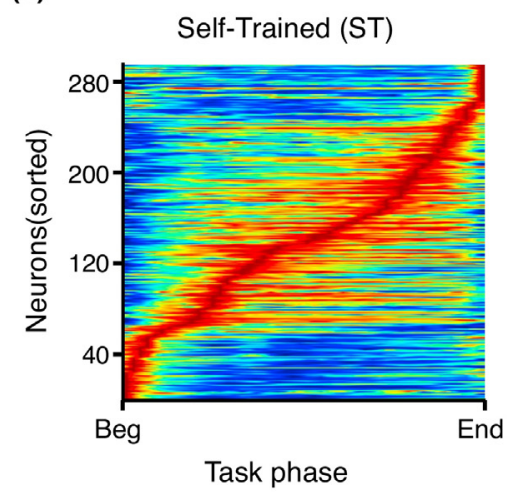

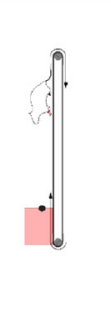
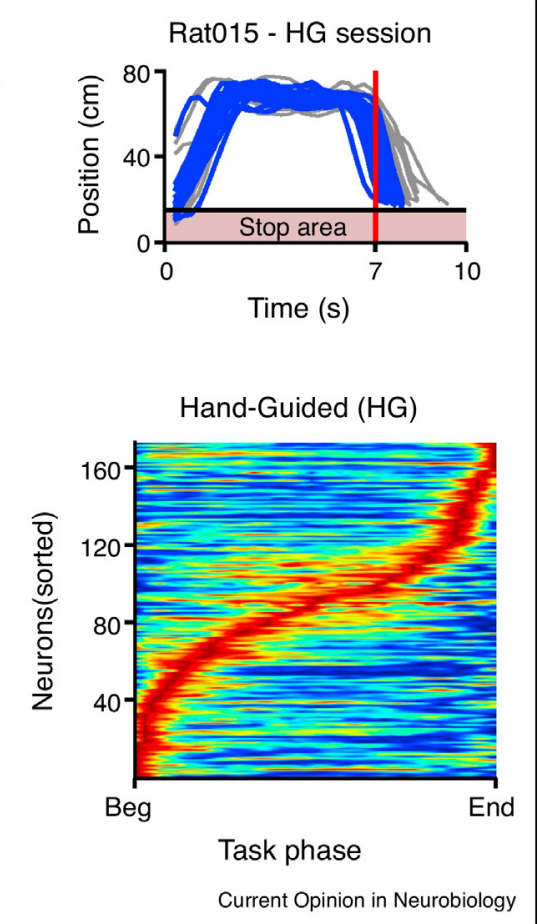

Over-representation of beginning and ending of a running sequence in naive and well-trained rats. (a) Rats trained in a time estimation task on a motorized treadmill developed a stereotyped back and forth running sequence (left). Hand-guided naive animals can perform a similar sequence (right) [50*]. Blue(gray) traces are trajectories for correct(incorrect) trials. (b) Normalized average firing rates (sorted according to the task phase of the maximum firing rate) of all the positively modulated neurons recorded in self-trained (left) and naive hand-guided animals (right, modified from [70]).

trial-and-error, the movement content and kinematics of a motor sequence, until it matches the time interval to estimate (which is typically associated with reward delivery). In one of the aforementioned time-estimation studies, a lesion of the primary motor cortex in naive rats prevented the learning of the embodied strategy but the same lesion was without effect when performed after learning. It was suggested that, during learning, the motor cortex provided a 'tutor' signal to subcorticals motor regions [60], most likely to the DLS [61]. In our general hypothesis, efference copy of motor programs and somatosensory responses would tutor DS SPN. During learning, when correct or incorrect behavioral sequences are performed, the opposite modulatory power of dopamine on dSPN and iSPN activity would facilitate and depress the cellular link between sensorimotor states and motor programs, respectively. In support of this hypothesis, when mice were given the possibility to self-stimulate their own dSPN by touching a capacitive switch, they quickly repeated such action. On the opposite a similar self-stimulation of iSPN induced avoidance of the switch [62]. Moreover, it was recently shown that the specific stimulation of dSPN or iSPN while mice performed a simple action at certain speed was sufficient to produce specific and sustained increases or decreases in the selection of this speed $\left[63^{\circ}\right]$. Altogether, these different studies support the idea that the DS, by combining continuous sensorimotor state estimation and bidirectional dopamine-based neuromodulation/plasticity, could contribute to learning, at the level of action content and kinematics. Such general function might apply to a wide range of behavioral context, that is beyond trial-and-error embodied strategy for time estimation.

\section{Conclusion and outlook}

Compelling evidence in support of prominent somatosensory responses in the dorsal striatum have been reviewed, along with the experimental challenges associated with such responses and their possible functional implication in trial-and-error motor learning. It is nevertheless important to highlight that DS neurons are not just sensitive to specific somatosensory stimuli. They can also integrate multimodal information. This has been elegantly demonstrated in single-cell recordings of SPN in anesthetized mice, in response to visual and whisker stimuli $\left[36^{\bullet \bullet}\right]$. Early electrophysiological experiments in behaving non-human primates had also shown that DS neurons respond to movements or sensory stimuli in a 
context-sensitive manner [4]. More recently, during time estimation tasks, the activity of individual DS neurons was reported to be comodulated by time and movementsrelated aspect of task performance $\left[50^{\circ}, 64^{\circ}\right]$. It has also been proposed that the striatum outperforms the prefrontal cortex in predicting elapsed time because it integrates incoming information from multiple cortical areas [65]. Two important questions need to be addressed in the future regarding the integrative capacity of the striatum. First, how much of this integration is performed by DS neurons versus conveyed by external input? Indeed, while the DS receives inputs from a number of functionally distinct brain regions $\left[32^{\bullet \bullet}, 33^{\bullet \bullet}\right]$, these inputs might already convey integrated information, as illustrated in the visual cortex with the robust modulation of visually evoked responses by locomotion [66]. Second, the relative contribution of cortical and thalamic input to striatal patterns of activity during behavior needs to be further specified. While the predominance of the cortical contribution to whisker-evoked striatal responses has been recently demonstrated [67], there is also converging evidence that thalamic input contribute to the processing of somatosensory and motor information in the striatum $\left[32^{\bullet \bullet}, 68\right]$. It has been proposed that, to support motor learning, striatal neurons may integrate complementary information provided by the cortex and thalamus [69]. Progress in our understanding of the striatum function will require to test the predictions of such precise models [69] and to address the aforementioned gaps in knowledge, all this while keeping in mind that, even in the BG, modulations of patterns of neuronal activity that follow movements are as interesting than those preceding them.

\section{Conflict of interest statement}

Nothing declared.

\section{Acknowledgements}

DR work is supported by a European Research Council (ERC-2013CoG - 615699_NeuroKinematics, D.R.). The author would like to thank Dr Ingrid Bureau and Mostafa Safaie for critical reading of the manuscript and past and current team members for stimulant discussions.

\section{References and recommended reading}

Papers of particular interest, published within the period of review, have been highlighted as:

- of special interest

$\bullet$ of outstanding interest

1. Redgrave $\mathrm{P}$ et al.: Goal-directed and habitual control in the basal ganglia: implications for Parkinson's disease. Nat Rev Neurosci 2010, 11:760-772.

2. Dudman JT, Krakauer JW: The basal ganglia: from motor commands to the control of vigor. Curr Opin Neurobiol 2016, 37:158-166.

3. Turner RS, Desmurget M: Basal ganglia contributions to motor control: a vigorous tutor. Curr Opin Neurobiol 2010, 20:1-13.

4. Mink JW: The basal ganglia: focused selection and inhibition of competing motor programs. Prog Neurobiol 1996, 50:381-425.
5. Redgrave P, Prescott TJ, Gurney K: The basal ganglia: a vertebrate solution to the selection problem? Neuroscience 1999, 89:1009-1023.

6. Turner RS, Pasquereau B: In The Cognitive Neuroscience. Edited by Gazzaniga M, Mangun G. The MIT Press; 2014:452-467.

7. Deniau J-M, Mailly P, Maurice N, Charpier S: The pars reticulata of the substantia nigra: a window to basal ganglia output. Prog Brain Res 2007, 160:151-172.

8. Hikosaka O, Takikawa Y, Kawagoe R: Role of the basal ganglia in the control of purposive saccadic eye movements. Physiol Rev 2000, 80:953-978.

9. Kreitzer AC, Malenka RC: Striatal plasticity and basal ganglia circuit function. Neuron 2008, 60:543-554.

10. Kravitz AV et al.: Regulation of parkinsonian motor behaviours by optogenetic control of basal ganglia circuitry. Nature 2010 466:622-626.

11. Chevalier G, Deniau JM: Disinhibition as a basic process in the expression of striatal functions. Trends Neurosci 1990, 13:277 280.

12. Wolpert DM, Ghahramani Z, Flanagan JR: Perspectives and problems in motor learning. Trends Cogn Sci 2001, 5:487-494.

13. Künzle $\mathrm{H}$ : An autoradiographic analysis of the efferent connections from premotor and adjacent prefrontal regions (Areas 6 and 9) in Macaca fascicularis. Brain Behav Evol 1978 15:201-234.

14. Künzle $\mathrm{H}$ : Bilateral projections from precentral motor cortex to the putamen and other parts of the basal ganglia. An autoradiographic study in Macaca fascicularis. Brain Res 1975 88:195-209.

15. McGeorge AJ, Faull RL: The organization of the projection from the cerebral cortex to the striatum in the rat. Neuroscience 1989, 29:503-537.

16. Parent A, Hazrati L-N: Functional anatomy of the basal ganglia I. The cortico-basal ganglia-thalamo-cortical loop. Brain Res Rev 1995, 20:91-127.

17. Alexander GE, DeLong MR, Strick PL: Parallel organization of functionally segregated circuits linking basal ganglia and cortex. Annu Rev Neurosci 1986, 9:357-381.

18. Haber SN: The primate basal ganglia: parallel and integrative networks. J Chem Neuroanat 2003, 26:317-330.

19. Flaherty AW, Graybiel AM: Motor and somatosensory corticostriatal projection magnifications in the squirrel monkey. J Neurophysiol 1995, 74:2638-2648.

20. Flaherty AW, Graybiel AM: Input-output organization of the sensorimotor striatum in the squirrel monkey. J Neurosci 1994, 14:599-610.

21. Flaherty AW, Graybiel AM: Corticostriatal transformations in the primate somatosensory system. Projections from physiologically mapped body-part representations. J Neurophysiol 1991, 66:1249-1263.

22. Vertes RP, Hoover WB, Szigeti-buck K, Leranth C: Nucleus reuniens of the midline thalamus: link between the medial prefrontal cortex and the hippocampus. Brain Res Bull 2007, 71:601-609.

23. Hoover JE, Hoffer ZS, Alloway KD: Projections from primary somatosensory cortex to the neostriatum: the role of somatotopic continuity in corticostriatal convergence. $J$ Neurophysiol 2003, 89:1576-1587.

24. Carelli RM, West MO: Representation of the body by single neurons in the dorsolateral striatum of the awake, unrestrained rat. J Comp Neurol 1991, 309:231-249.

25. Mittler T, Cho J, Peoples LL, West MO: Representation of the body in the lateral striatum of the freely moving rat: single neurons related to licking. Exp Brain Res 1994, 98: 163-167. 
26. West $\mathrm{MO}$ et al.: A region in the dorsolateral striatum of the rat exhibiting single-unit correlations with specific locomotor limb movements. J Neurophysiol 1990, 64:1233-1246.

27. Cho J, West MO: Distributions of single neurons related to body parts in the lateral striatum of the rat. Brain Res 1997 756:241-246.

28. Oran Y, Bar-Gad I: Loss of balance between striatal feedforward inhibition and corticostriatal excitation leads to tremor. J Neurosci 2018, 38:2821-2917.

29. Hoffer ZS, Alloway KD: Organization of corticostriatal projections from the vibrissal representations in the primary motor and somatosensory cortical areas of rodents. J Comp Neurol 2001, 439:87-103.

30. Alloway KD, Mutic JJ, Hoffer ZS, Hoover JE: Overlapping corticostriatal projections from the rodent vibrissal representations in primary and secondary somatosensory cortex. J Comp Neurol 2000, 426:51-67.

31. Pan WX, Mao T, Dudman JT: Inputs to the dorsal striatum of the mouse reflect the parallel circuit architecture of the forebrain. Front Neuroanat 2010, 4:1-14.

32. Hunnicutt BJ et al: : A comprehensive excitatory input map of - $\quad$ the striatum reveals novel functional organization. Elife 2016, 5:e19103.

See annotation to $\left[33^{\circ}\right]$.

33. Hintiryan $\mathrm{H}$ et al:: The mouse cortico-striatal projectome. Nat -• Neurosci 2016, 19:1100-1114.

These studies used computational neuroanatomic approaches to quantify, in the striatum, the anatomical labeling resulting from several hundreds of well-localized single, double and triple injections of anterograde tracers in different regions of the cortex and thalamus of mice. A detailed quantitative information on the organization of excitatory inputs on the striatum is provided. In regard of the present review, the importance of sensorimotor input to the striatum, beyond the classical dorsolateral limit, is remarkable. These studies also provided quantitative evidence for convergence between functionally related sensory and motor cortical areas and delineated functional territories related to distinct body parts.

34. Mowery TM, Harrold JB, Alloway KD: Repeated whisker stimulation evokes invariant neuronal responses in the dorsolateral striatum of anesthetized rats: a potential correlate of sensorimotor habits. J Neurophysiol 2011, 105:2225-2238.

35. Pidoux M, Mahon S, Deniau J-M, Charpier S: Integration and propagation of somatosensory responses in the corticostriatal pathway: an intracellular study in vivo. J Physiol 2011, 589:263-281.

36. Reig R, Silberberg G: Multisensory integration in the mouse -• striatum. Neuron 2014, 83:1200-1212.

The authors performed whole-cell recordings of identified SPN in responses to unilateral and bilateral whisker stimulation and found that DLS SPN, but not cortical neurons, integrated bilateral whisker stimulation. They also reported that DMS neurons could integrate multimodal (visual and tactile) information.

37. Nagy A, Eördegh G, Paróczy Z, Márkus Z, Benedek G: Multisensory integration in the basal ganglia. Eur $J$ Neurosci 2006, 24:917-924.

38. Ketzef $\mathrm{M}$ et al.: Dopamine depletion impairs bilateral sensory

- $\quad$ processing in the striatum in a pathway-dependent manner. Neuron 2017, 94 855-865.e5.

In this follow-up study of Reig and Silberberg[36 $\left.6^{\circ}\right]$, the authors found that bilateral integration of whisker stimuli is impaired in dopamine depleted animals and is restored following L-Dopa treatment.

39. Conte A, Khan N, Defazio G, Rothwell JC, Berardelli A: Pathophysiology of somatosensory abnormalities in Parkinson disease. Nat Rev Neurol 2013, 9:687-697.

40. Sippy T, Lapray D, Crochet S, Petersen CCH: Cell-type-specific -• sensorimotor processing in striatal projection neurons during goal-directed behavior. Neuron 2015, 88:298-305.

The authors trained mice to lick a reward spout in response to single whisker deflections and performed whole-cell recordings and optogenetic stimulation of identified SPN. The results obtained suggest that the rapid response of direct pathway SPN to whisker deflection is critical for the expression of conditional licking response.
41. Panigrahi B et al.: Dopamine is required for the neural representation and control of movement vigor. Cell 2015, 162:1418-1430.

42. Barnes TD, Kubota Y, Hu D, Jin DZ, Graybiel AM: Activity of striatal neurons reflects dynamic encoding and recoding of procedural memories. Nature 2005, 437:1158-1161.

43. Jog MS, Kubota Y, Connolly Cl, Hillegaart V, Graybiel AM: Building neural representations of habits. Science 1999, 286:1745-1749.

44. Jin X, Costa RM: Start/stop signals emerge in nigrostriatal circuits during sequence learning. Nature 2010, 466:457-462.

45. Jin X, Tecuapetla F, Costa RM: Basal ganglia subcircuits distinctively encode the parsing and concatenation of action sequences. Nat Neurosci 2014, 17:423-430.

46. Cui G et al.: Concurrent activation of striatal direct and indirect pathways during action initiation. Nature 2013, 494:238-242.

47. Coffey KR, Nader M, Bawa J, West MO: Homogeneous

-. processing in the striatal direct and indirect pathways: single body part sensitive type Ilb neurons may express either dopamine receptor D1 or D2. Eur J Neurosci 2017, 46: 2380-2391.

The authors used optrodes to d/iSPN neurons and recorded their responses to various types of stimulation (e.g. cutaneous touch, passive manipulation, and active movements) of most of the accessible body parts (head, vibrissae, paw, chest, chin, snout, ear, shoulder, cheek pad, and trunk). They found that a large fraction of both $\mathrm{d} / \mathrm{iSPN}$ responded to passive stimulation of specific part of the body and that neurons sensitive to a given body part were close to each other.

48. Kim N, Barter JW, Sukharnikova T, Yin $\mathrm{HH}$ : Striatal firing rate reflects head movement velocity. Eur J Neurosci 2014, 40: 3481-3490.

49. Tang C, Pawlak AP, Prokopenko V, West MO: Changes in activity of the striatum during formation of a motor habit. Eur $\mathrm{J}$ Neurosci 2007, 25:1212-1227.

50. Rueda Orozco PE, Robbe D: The striatum multiplexes

- contextual and kinematic information to constrain motor habits execution. Nat Neurosci 2015, 18:453-460.

The authors used behavioral paradigm in which both naive and trained rats performed a running sequence with similar kinematics. In the DLS of trained animals, tetrode recorded revealed integrative representations of running speed, position and time. Still naive and trained rats displayed similar sequential population activity during runs suggesting a significant contribution of somatosensory responses to these sequential patterns.

51. Shi LH, Luo F, Woodward DJ, Chang JY: Neural responses in multiple basal ganglia regions during spontaneous and treadmill locomotion tasks in rats. Exp Brain Res 2004, 157: 303-314.

52. Klaus A et al.: The spatiotemporal organization of the striatum article the spatiotemporal organization of the striatum encodes action space. Neuron 2017, 95 1171-1180.e7.

53. Barbera G et al: : Spatially compact neural clusters in the dorsal striatum encode locomotion relevant information. Neuron 2016, 92:202-213.

54. Yamin HG, Stern EA, Cohen D: Parallel processing of environmental recognition and locomotion in the mouse striatum. J Neurosci 2013, 33:473-484.

55. Costa RM, Cohen D, Nicolelis MAL: Differential corticostriatal plasticity during fast and slow motor skill learning in mice. Curr Biol 2004, 14:1124-1134.

56. Martiros N, Burgess AA, Graybiel AM: Inversely active striatal projection neurons and interneurons selectively delimit useful behavioral sequences. Curr Biol 2018:560-573.

57. Rothwell PE et al.: Input- and output-specific regulation of serial order performance by corticostriatal circuits. Neuron 2015, 88:345-356.

58. Isomura $\mathrm{Y}$ et al.: Reward-modulated motor information in identified striatum neurons. J Neurosci 2013, 33:10209-10220.

59. Gouvêa TS et al:: Ongoing behavior predicts perceptual report of interval duration. Front Neurorobot 2014, 8:10. 
60. Kawai R et al:: Motor Cortex Is Required for Learning But Not for Executing a Motor Skill Article Motor Cortex Is Required for Learning But Not for Executing a Motor Skill. 2015:1-13 http://dx. doi.org/10.1016/j.neuron.2015.03.024.

61. Wolff SBE, Dhawale AK, Ko R, Ölveczky BP: Distinct roles for cortico- and thalamostriatal projections in motor skill learning and execution. Society for Neuroscience Annual Meeting 690.10/ JJ12 2017

62. Kravitz AV, Tye LD, Kreitzer AC: Distinct roles for direct and indirect pathway striatal neurons in reinforcement. Nat Neurosci 2012, 15:816-818.

63. Yttri EA, Dudman JT: Opponent and bidirectional control of

- movement velocity in the basal ganglia. Nature 2016:1-16 http:// dx.doi.org/10.1038/nature17639.

The authors stimulated dSPN and iSPN while head-restrained mice pressed on a bar. The stimulation was only preformed on trials initiated in a given range of speeds. Stimulation of the dSPN biased the subsequent animal choices toward the stimulated speed range. Stimulation of the iSPN biased the animal speed choices away from the stimulated speed range.

64. Mello GBMM, Soares S, Paton JJ: A scalable population code - for time in the striatum. Curr Biol 2015, 25:1113-1122.
The authors recorded spiking activity in the DS while rats performed a lever-press interval timing task. They found that striatal neurons activity integrated both task-relevant timing and sensorimotor information.

65. Bakhurin $\mathrm{KI}$ et al.: Differential encoding of time by prefrontal and striatal network dynamics. J Neurosci 2016, 37:854-870.

66. Niell CM, Stryker MP: Modulation of visual responses by behavioral state in mouse visual cortex. Neuron 2010, 65 472-479.

67. Reig R, Silberberg G: Distinct corticostriatal and intracortical pathways mediate bilateral sensory responses in the striatum. Cereb Cortex 2016, 26:4405-4415.

68. Alloway KD, Smith JB, Mowery TM, Watson GDR: Sensory processing in the dorsolateral striatum: the contribution of thalamostriatal pathways. Front Syst Neurosci 2017, 11:1-19.

69. Fee MS: Oculomotor learning revisited: a model of reinforcement learning in the basal ganglia incorporating an efference copy of motor actions. Front Neural Circuits 2012 6:38.

70. Taouali W, Rueda-Orozco PE, Robbe D: A minority-ruled population coding of kinematics in the striatum. bioRxiv 2017:130237 http://dx.doi.org/10.1101/130237. 